\title{
Deutsche Ärzte sind besonders attraktiv
}

\author{
Chefredakteur Dr. med. Dirk Einecke
}

\section{GEK REPORT}

\section{Deutsche gehen besonders oft zum Arzt}

Durchschnittlich 16,3-mal im Jahr suchen die Deutschen einen Arzt auf. Das hat die Auswertung der Daten von 1,5 Millionen Versicherten der Gmünder Ersatzkasse (GEK) aus dem Jahr 2004 ergeben. Damit belegen die Deutschen im internationalen Vergleich einen Spitzenplatz, so der letzte Woche veröffentlichte GEK Report. Dänen, Franzosen und Italiener zum Beispiel gehen nur sechs- bis achtmal im Jahr zum Arzt. Ähnlich hohe Arztkontaktzahlen wie in Deutschland werden dagegen aus Tschechien und Japan berichtet.

Besonders beliebter Tag für den Praxisbesuch - richtig, das ist der Montag (siehe Abbildung).

Vor allem von jungen Männern werden Ärzte eher gemieden (siehe MMW-Report): Bis zum 40. Lebensjahr lassen sie sich dort nur halb so oft sehen wie gleichaltrige Frau-

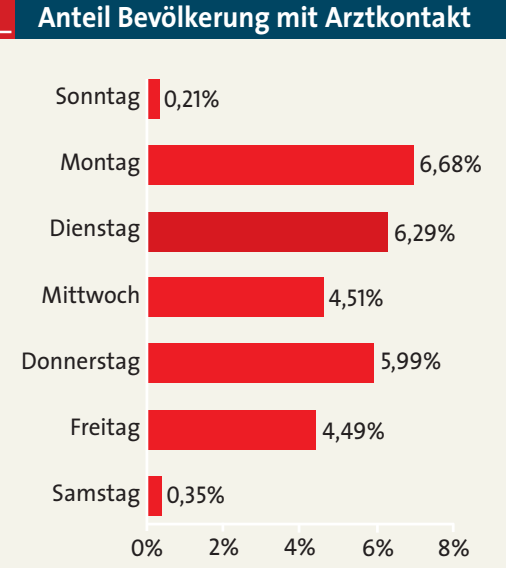

Zu Wochenbeginn versammeln sich die meisten Patienten in den Arztpraxen.

en. Erst ab dem 75. Lebensjahr ist die Zahl der Arztbesuche geschlechtsunabhängig.

GEK Report ambulant-ärztliche Versorgung

\section{TENNISELLENBOGEN}

\section{Physiotherapie schlägt Cortison im Langzeitverlauf}

In einer randomisierten Studie wurden 198 Patienten mit Epicondylitis humeri lateralis mit drei verschiedenen Methoden behandelt: Steroidinjektionen, Physiotherapie oder eine abwartende Strategie. Alle Patienten konnten nicht steroidale Antirheumatika bei Bedarf nehmen. Die Steroidinjektionen ergaben zwar nach sechs Wochen die besten Ergebnisse, in $72 \%$ der Fälle kam es jedoch da- nach zu einem Rückfall. Abwarten und Krankengymnastik schnitten langfristig ( 52 Wochen) in etwa gleich gut ab, in den ersten sechs Wochen war die Physiotherapie überlegen. Die Patienten, die krankengymnastisch behandelt worden waren, brauchten insgesamt signifikant weniger Schmerzmittel als die Patienten der beiden anderen Gruppen.

BMJ 2006;333:939-41

\section{Zitat der Woche}

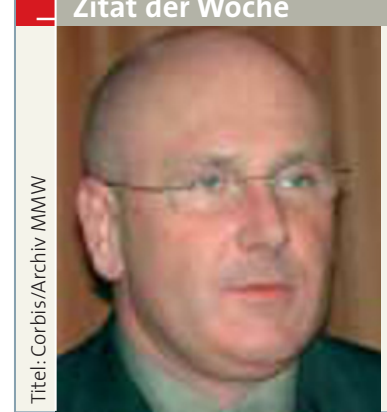

„Falls unsere Warnungen nicht gehört werden, werden die Verantwortlichen bald merken: Am Ende wird der Patient immer vom Arzt behandelt, nicht von den Politikern."

Dr. Andreas Köhler, Vorstandsvorsitzender der KBV auf der gemeinsamen Pressekonferenz der Spitzenverbände der gesetzlichen Krankenkassen, der KBV und der DKG am 6.11.2006 in Berlin

\section{STÜRZE IM ALTER}

\section{Mit dem Testosteron fällt der ganze Mann}

Alte Männer, deren Testosteronwert besonders niedrig ist, haben ein signifikant höheres Sturzrisiko als Gleichaltrige mit höheren Hormonspiegeln. Dies ergab eine Kohortenstudie mit 2587 Männern im Alter von 65-99 Jahren. Die Probanden wurden vier Jahre lang beobachtet. Die Männer mit Testosteronwerten in der untersten Quartile hatten im Vergleich mit den Probanden mit den höchsten Werten ein um 40\% erhöhtes Sturzrisiko. Der Effekt war bei den jüngeren Senioren (65-69 Jahre) am ausgeprägtesten. Arch Intern Med 2006;166:2124-31

\section{WIRKSAMES MITTEL GEGEN MÜDIGKEIT}

\section{Sport macht munter}

Müde und kaputt von einem langen Praxistag? Dann sollten Sie Sport machen! Auch wenn körperliche Aktivität vielleicht das Letzte ist, worauf Sie jetzt noch Lust haben - gegen die Müdigkeit hilft sie. Das zeigt eine Metaanalyse von 70 Studien mit fast 7000 Patienten: Egal ob die Studienteilnehmer körperlich gesund oder chronisch krank waren, fühlten sie sich weniger müde und hatten mehr Energie, wenn sie regelmäßig Sport trieben. J Psych Bulletin, Nov 2006

\section{KOHLENHYDRAT-ARME DIÄTEN}

\section{Sicher für das Herz}

Kohlenhydratarme Diäten scheinen das KHK-Risiko bei Frauen nicht zu erhöhen. Dies wurde jetzt in der Nurses' HealthStudie mit 82802 Teilnehmerinnen festgestellt. Dabei waren die Ernährungsgewohnheiten mittels Fragebogen erfasst und mit dem KHK-Risiko im 20-jährigen Verlauf in Beziehung gesetzt worden. Die Autoren weisen allerdings darauf hin, dass sich die Studie auf allgemeine Ernährungsgewohnheiten bezog und nicht eine bestimmte Diätform, z. B. die proteinund fettreiche Atkinsdiät, untersuchte. N Engl J Med 2006;355:1991-2002 